\title{
Sistemas de información geográfico en el manejo de los servicios básicos de salud en el municipio de San José Colinas, Santa Bárbara, Honduras
}

Yeny Maribel Castellanos

\section{Resumen}

Como parte de la generación y transmisión del conocimiento, los sistemas de información geográfica ofrecen los medios necesarios para llevar a cabo una diversidad de investigaciones, en este caso el manejo de los servicios básicos de salud en el municipio de San José Colinas, Santa Bárbara, en donde la necesidad de precisar la satisfacción de la población del municipio respecto al acceso a estos servicios de salud tanto en áreas rurales como urbanas, en donde la importancia de identificar los lugares que brindan una atención primaria de salud, y las condiciones de acceso a estos centros de salud sean las adecuadas en este municipio. Con la identificación y localización de la información cartográfica que posea el municipio será un factor determinante no solo en la promoción de los sistemas de información geográfica enfocada a la salud, sino también generar una visión amplia en el desarrollo de proyectos que permita potenciar el desarrollo de la zona, en donde esta visión podrá mejorar áreas como catastro, servicios básicos, manejo y uso de suelo, manejo de recurso naturales, turismo, infraestructura u otro que se desee implementar en la región.

Palabras clave: Sistemas de información geográfica, accesos a servicios básicos, métodos, territorio, tecnologías de la información geográfica.

\section{Abstract}

In recent years, the Municipality of San Jose Hills, Santa Barbara presents an increase of requests for implementation of plans and projects for the municipality in all areas. Which is why we need to identify the geographic and demographic characteristics of the municipality (age, gender, family background) necessary or missing in geospatial data to balance the implementation of basic services as part 
of the satisfaction of human needs and is in where with the help of the method and techniques of Geographic Information Systems (GIS) will be a contribution to the improvement processes for the development of the municipality. When talking about the inequalities of basic services are primary care problems, which should be minimized by local authorities. Reason why it is necessary to develop the methodology in various phases Phase I-Assessment of the situation, which identifies existing problems based on basic services; Phase II Study of needs and design of space demand, is where a detailed examination of the factors that have influenced achieve satisfaction or dissatisfaction of the affected population, Phase III-distribution schemes Formulation of spatial functioning equipment and services that help display the status of the population in relation to basic services where the diagnosis will help us identify the existing problems. Phase IV-Decisions will be designed from the findings, Phase V-Evaluation of achievements, where we determine that decisions achieving targets. As results have identified areas of study areas in the Municipality of San Jose Hills, Santa Barbara, with the collaboration of the University Liaison Address. Tabulated already $60 \%$ of the data.

Key words: Basic services, methods, techniques, GIS

Yeny Maribel Castellanos (Yeny.castell@gmail.com) Departamento de Ciencia y Tecnologías de la Información Geográfica, Facultad de Ciencias Espaciales, Universidad Nacional Autónoma de Honduras. 


\section{Introducción}

La importancia de los servicios colectivos para la vida humana resulta fuera de toda duda y ello puede ser constatado, entre otros, por varios hechos meridianos. En primer lugar, se puede apreciar que la satisfacción de muchas necesidades humanas, algunas de ellas básicas, se realiza mediante servicios que son dispensados en los denominados equipamientos. Nuestra existencia cotidiana se nos aparece así soportada por una inmensa gama de servicios y resulta una tendencia temporal incontestable que el ciudadano demanda una cifra creciente de ellos. En segundo lugar, ello se vincula con el hecho de que en los países desarrollados la prioridad conferida a los mismos haya ido logrando un elevado grado de dotación y calidad de los mismos, lo que expresa un esfuerzo económico y una dedicación de recursos humanos muy notables. (Anselin, 2003)

Actualmente estas técnicas se potencian al contar con las posibilidades de vinculación interactivas entre las representaciones cartográficas digitales y las bases de datos asociadas en el ambiente de los SIG y los Sistema de Apoyo a las Decisiones Espaciales -SADE (Anselin, 2003), avanzando en los caminos que propone la Visualización Geográfica (GVis, Geographical Visualization) como nuevo campo de desarrollo temático.

\section{Metodología}

Se considera la organización de las tareas en fases como una guía que se adapte a las particularidades de la investigación. Como guía metodológica se ha tomado del estudio desarrollado por Antonio Moreno, el cual fue desarrollado en fases que se describen a continuación: a) Diagnostico situacional, en donde se identificó las zonas de estudio, b) Identificación de inventario de los equipamientos, de los recursos disponibles y de sus atributos. c) Base de datos con las delimitaciones de las áreas de servicio actuales y sus atributos, zonas escolares, áreas de salud, áreas de servicios sociales, etc. d) Estudio de necesidades y dimensionamiento de la demanda espacial. e) Análisis de la accesibilidad espacial actual a los puntos de servicios desde cada lugar con demanda. f) Visualización exploratoria de datos y presentación cartográfica de resultados. g) Formulación de esquemas de distribución de los equipamientos y funcionamiento espacial de los servicios. h) Definición de los patrones espaciales de la oferta, de dotaciones, y qué forma de funcionamiento espacial de los servicios provistos resulta más apropiada, técnicas como los modelos de localización óptima. 


\section{Análisis}

Para la identificación de la Zona de Estudio y la organización de los datos, se tomó en cuenta el inventario existente de los equipamientos con sus atributos tomado del Sistema Nacional de Información Territorial (SINIT), y del Instituto Nacional de Estadística del 2001, (INE2001).

En la organización se descartaron los datos que no favorecían en el análisis de la información.

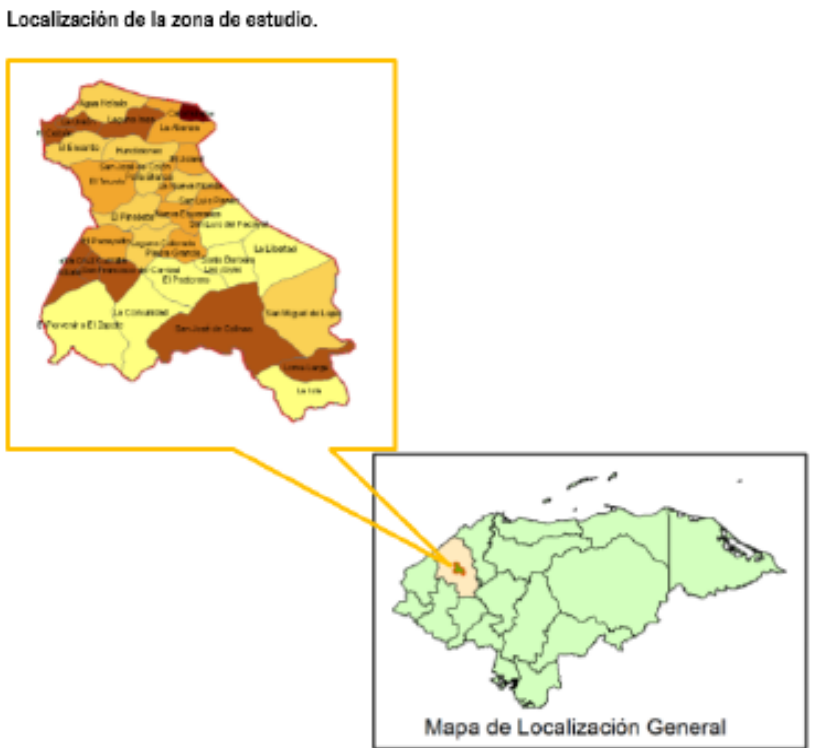

Figura 1. Identificación del Área de Estudio

\begin{tabular}{|c|l|c|c|}
\hline \multicolumn{5}{|c|}{ San José de las Colinas (coordenadas) } \\
\hline Coordenadas & $15^{\circ} 1^{\prime} 59.99^{\prime \prime} \mathrm{N}, 8^{\circ} 18^{\prime} 0^{\prime \prime} \mathrm{W}$ & En decimal & $15.03333^{\circ},-88.3^{\circ}$ \\
\hline UTM & $166242436025216 \mathrm{P}$ & & \\
\hline Zoom & 6 & Escala & $\pm 1: 100000$ \\
\hline Región & & Tipo & City \\
\hline
\end{tabular}

Cuadro 1. Coordenadas de la Zona de Estudio 
Utilizando la base de datos con las delimitaciones de las áreas de servicio actuales y sus atributos, que describen las diferentes áreas de servicios básicos y los propios de la zona, permite visualizar la importancia de los sistemas de información geográfica aplicados a los territorios reales.

En donde la identificación de las zonas geográfica puede potenciar las comunidades que posee limitaciones, trabajando con las capacidades de las zonas más fuertes, esto beneficiaría todo el municipio.
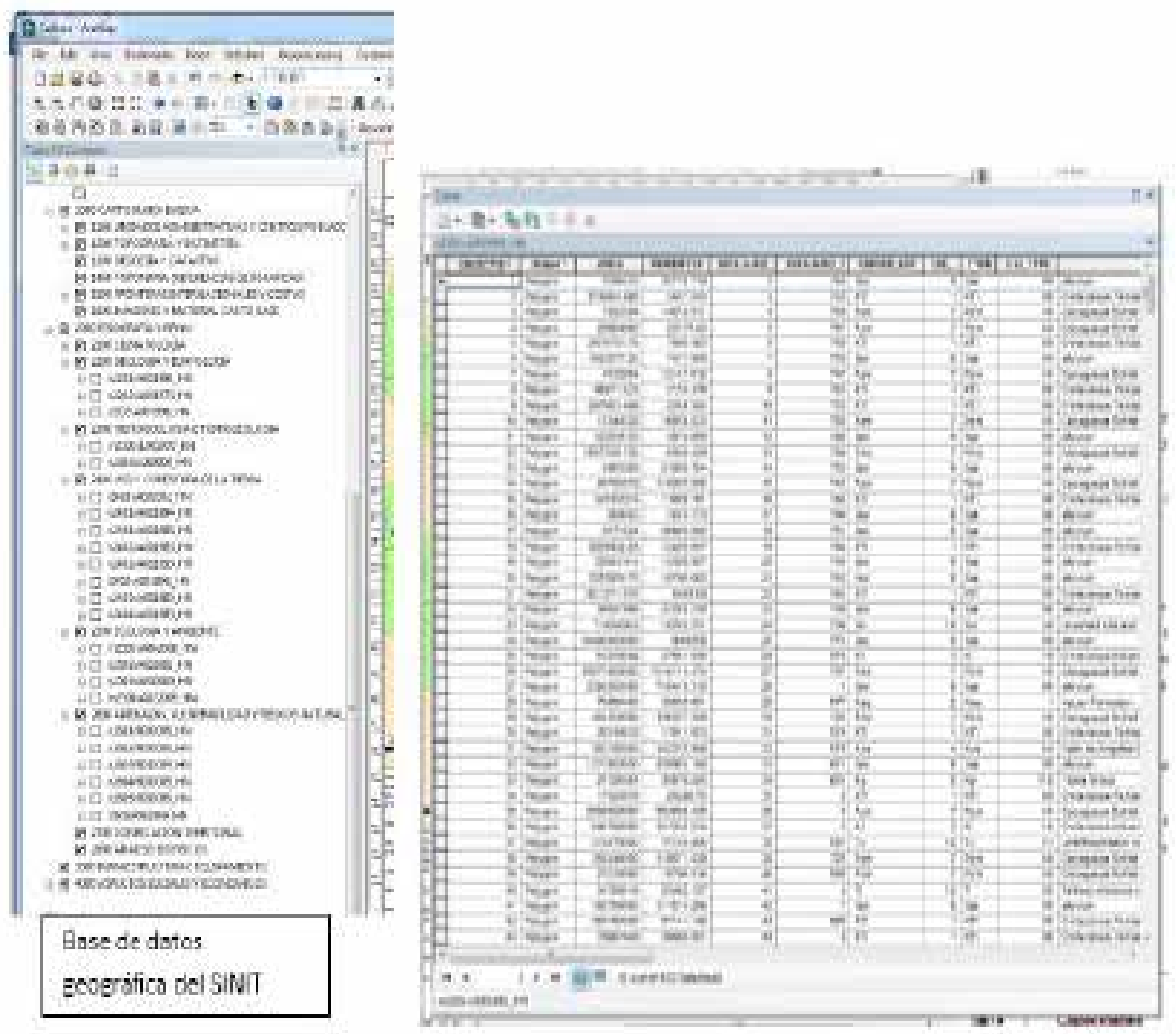

Figura 2. Data utilizada para el análisis

\section{Resultados}

Con el XVI Censo de Población y V de Vivienda del 2001, se ha logrado utilizar los datos producidos en el INE, para organizar las bases de datos espaciales, 
y así mostrar los rasgos más relevantes de esta población, en donde con el uso de las geo data bases se logra identificar la infraestructura y medios para el transporte (vías -calles, carreteras, etc.-, líneas de transporte, paradas y estaciones, etc.)

Se muestra la distribución de la Infraestructura existente en el Municipio de San José Colinas en relación a los asentamientos humanos, mostrando así su crecimiento, concentrándose en las cabeceras principales.

\section{Infraestructura - Aldeas San José Colinas Departamento de Santa Bárbara}

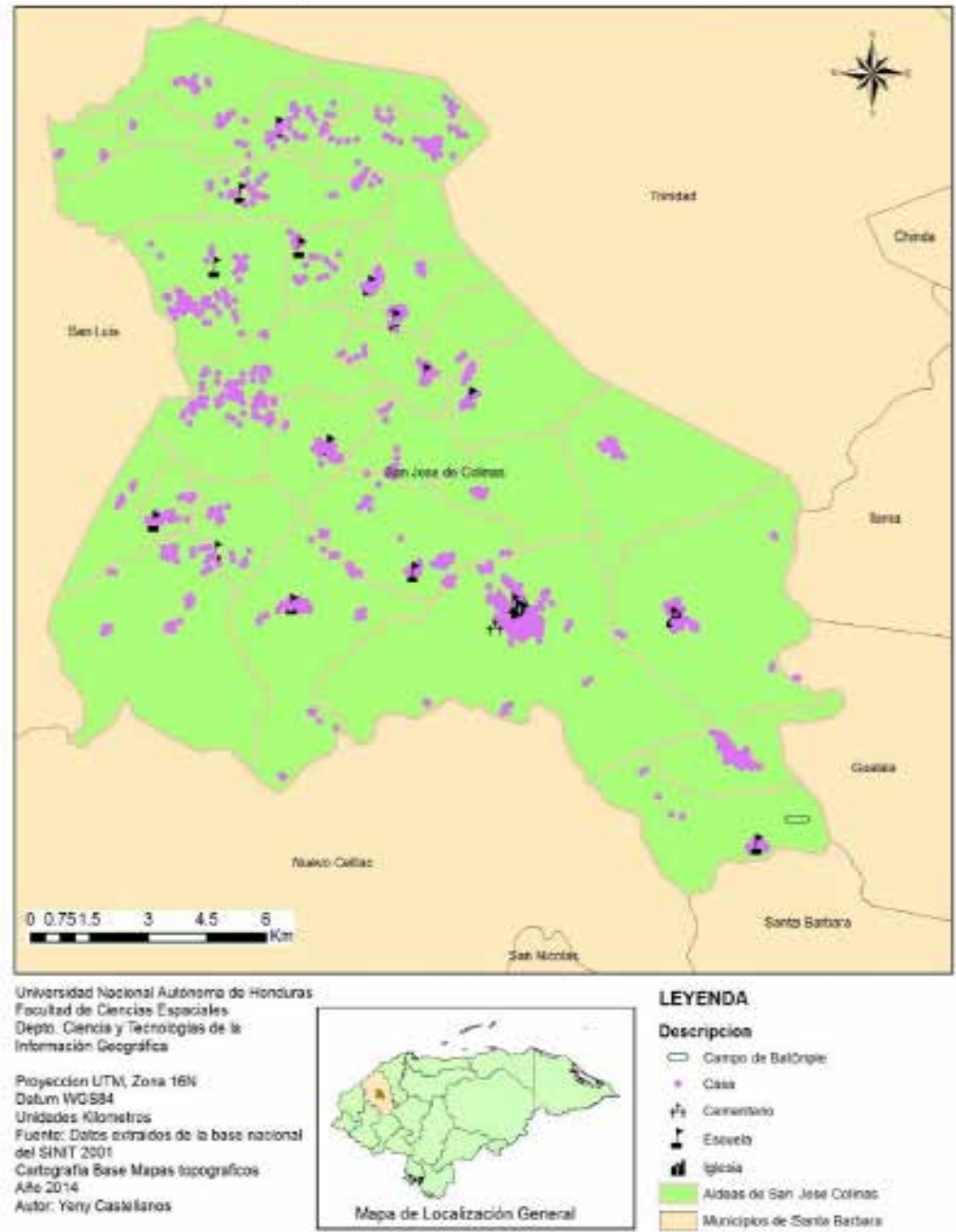

Figura 3. Mapa de Infraestructura 


\section{Servicios de Salud - Aldeas San José Colinas Departamento de Santa Bárbara}

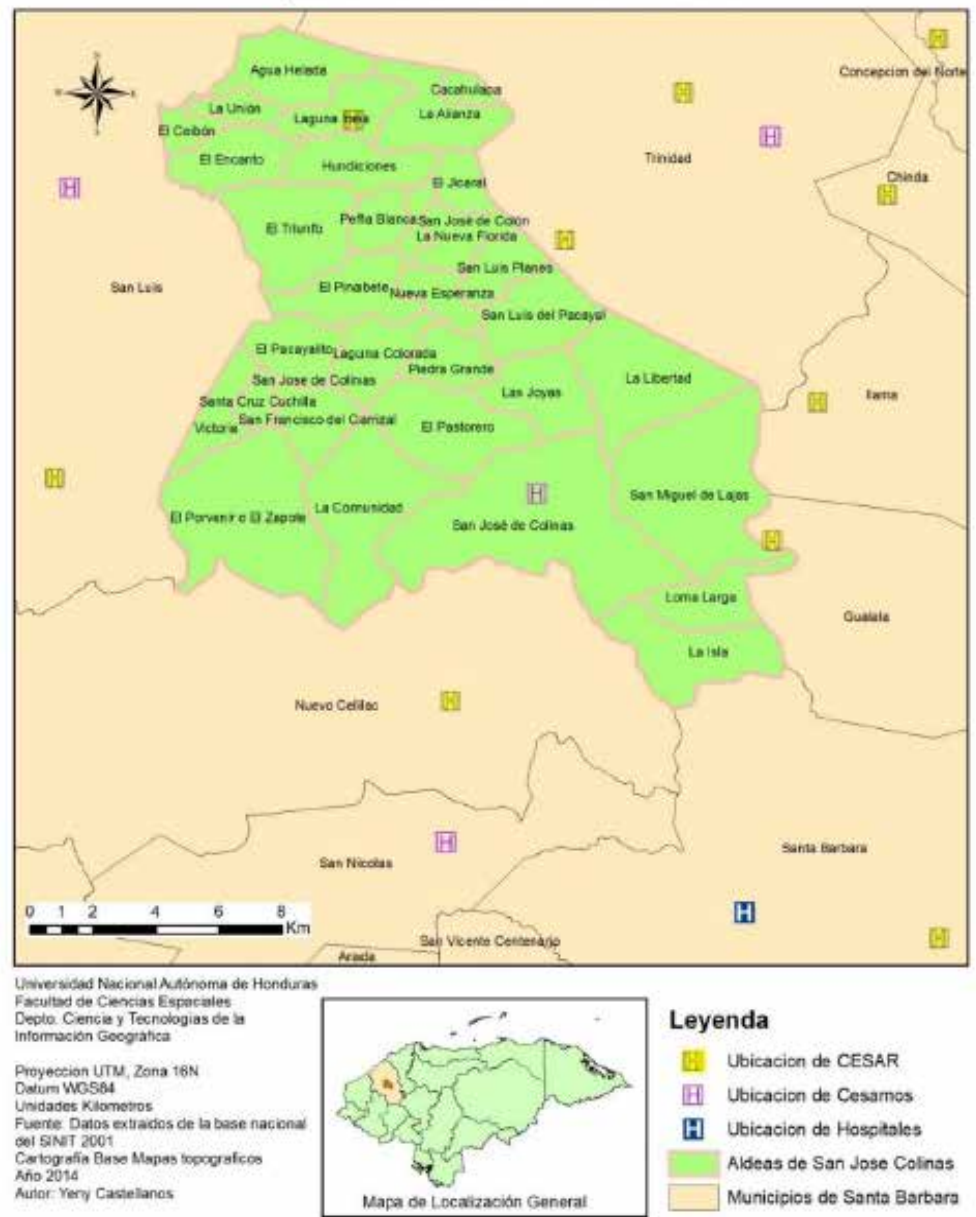

Figura 4.Mapa de Servicios de Salud

La distribución de los principales centros de atención en salud, se encuentra localizados de manera aislada, a los núcleos de población, haciendo notar la necesidad de potenciar esta necesidad, ya que por el crecimiento poblacional no se dará abasto suplir las exigencias en salud de este municipio.

Se puede observar que el Hospital principal de la zona se encuentra en el Municipio de Santa Bárbara, y en los últimos años su capacidad instalada no cubre la demanda de toda esa región por lo que es priorizar que el Cesamo que se 
encuentra en el Municipio de San José Colinas pueda considerar y reestructurar su categoría o evaluar su capacidad instalada para que amplíe sus servicios a las comunidades de las zonas aledañas.

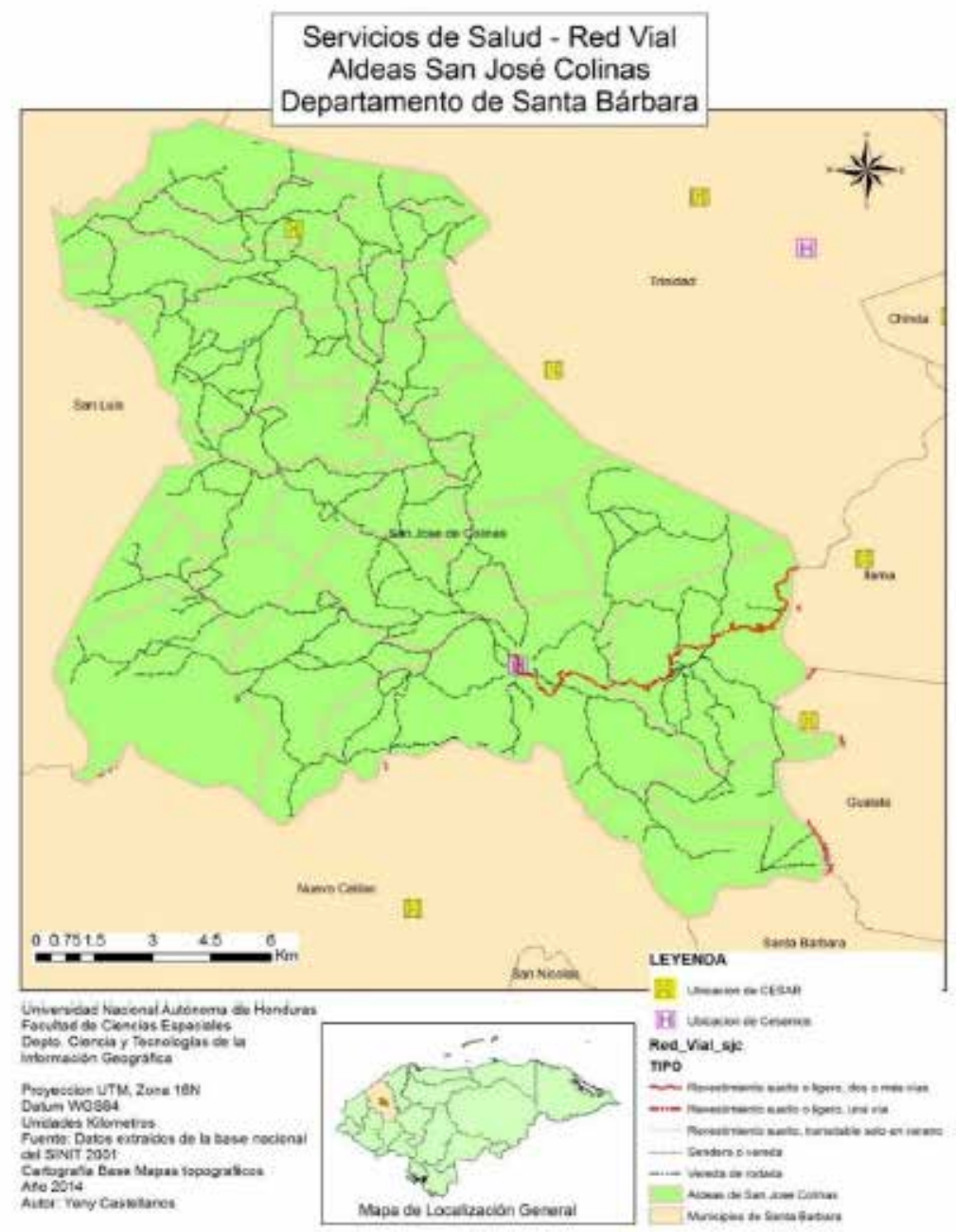

Figura 5. Mapa de Servicios de Salud-Red Vial

En relación a las vías de comunicación, no son las óptimas para los desplazamientos de los habitantes a los centros de atención. 


\section{Discusión y Conclusiones}

La identificación de las zonas geográfica puede potenciar las comunidades que posee limitaciones, trabajando con las capacidades de las zonas más fuertes, esto beneficiaría todo el municipio.

Como se observa en los datos depurados y representados visualmente, el Municipio de San José de Colinas cuenta con dos Cesamos y un Cesar, estos en su momento se encontraban distribuidos estratégicamente en la zona, pero demostrando que la cobertura geográfica en la actualidad está muy replegada del resto de las aldeas del municipio.

Con los datos presentados es necesario que se evalúe nuevamente la capacidad instalada de los centros de asistencia de salud nuevamente para las demandas actuales, por la tendencia de crecimiento de la población. De esta forma disminuiría el sobre cargo de la atención centralizada que existe del hospital general que se encuentra en la cabecera departamental de Santa Bárbara.

\section{Bibliografía}

- Buzai, G. B. (2011). Análisis Sociespacial con Sistemas de Información Geográfica. Buenos Aires: Lugar Editorial S. A.

- Buzai, G., Moreno, A., \& Baxendale, C. (2008). Análisis exploratorio de datos espaciales educativos: Aplicación a la Ciudad de Lujan. En C. Baxendale, L. Bevilaqua, G. Buzai, A. Moreno, A. Semorile, \& D. Valdez A., Análisis y Planificación de Servicios Colectivos con Sistemas de Información Geográfica (pág. 149). Madrid: Grafiprint S.L. .

- Gonzalez, M. S. (4,5 y 6 de Octubre de 2011). Foro Internacional MEDAMERICA 2011. Recuperado el septiembre de 2013, de Foro MEDAMERICA: http:// www.ub.edu/medame/foro_ptdr/presentaciones_11.htm

- Hein, A. (Junio de 2009). Fundación Paz Ciudadana. Recuperado el 2013, de La Georreferenciación como herramienta para el diagnóstico de problemas de seguridad ciudadana en el ámbito local: http://www.pazciudadana.cl/docs/ pub_20090623122857.pdf 
- Moreno, A. (2008). Los servicios colectivos y el desarrollo territorial. En G. Buzai, A. Moreno, \& C. Baxendale, Análisis y Planificación de Servicios Colectivos con Sistemas de Información Geográfica (pág. 149). Madrid: Grafiprint S. L.

- Pineda, E. A. (2008). Metodología de la Investigación. Washington, D. C.: PALTEX-OMS.

- Ramírez, M. L. (2003). Cálculo de medidas de accesibilidad geográfica, temporal y económica generadas mediante sistemas de información geográfica. Primer Congreso de la Ciencia Cartográfica. 